\title{
FORMATION OF TOPOLOGICAL DEFECTS DURING PREHEATING
}

\author{
S. KASUYA AND M. KAWASAKI \\ Institute for Cosmic Ray Research, University of Tokyo \\ Tanashi, Tokyo 188, Japan
}

Recent investigations revealed that the explosive decay of the inflaton field takes place in the first stage of reheating due to the effect of parametric resonance (Kofman et al., 1994). One of surprising possibilities of the nonthermal fluctuations (created particles) during preheating is the symmetry restoration which may lead to the formation of topological defects (Kofman et al., 1996; Tkachev, 1996).

We study in detail the dynamics of a scalar field $\Phi$ with potential $g\left(|\Phi|^{2}-\eta^{2}\right)^{2} / 2$ ( $g$ : self-coupling constant, $\eta$ : symmetry breaking scale) after inflation and make clear whether topological defects can ever be formed during preheating (Kasuya \& Kawasaki, 1997). In particular, we pay attention to GUT defects $\left(\eta \sim 10^{15} \mathrm{GeV}-10^{17} \mathrm{GeV}\right)$, and consider three types of fluctuations. The first one is produced due to parametric resonance, the second is due to the negative curvature of the potential, and the last is created during inflation. We search for the parameter region that non-thermal fluctuations of the scalar field produced through the parametric resonant decay of its homogeneous part do not lead to defect formation.

We find that the GUT topological defects are not formed if $\eta$ is larger than $10^{13} \mathrm{GeV}$ for the real scalar field, and $10^{14} \mathrm{GeV}$ for the complex field. The difference is because the complex field can decay into the Goldstone mode more rapidly. In either case, this region is rather wide, and the GUT defects are not produced after inflation.

\section{References}

Kasuya, S. and Kawasaki, M. (1997) hep-ph/9703354, to appear in Phys. Rev. D. Kofman, L., Linde, A.D. and Strarobinsky, A. (1994) Phys. Rev. Lett. 73, 3195. Kofman, L., Linde, A.D. and Strarobinsky, A. (1996) Phys. Rev. Lett. 76, 1011. Tkachev I.I, (1996) Phys. Lett. B376, 35. 